\title{
Tully-Fisher Relations from an HI-Selected Sample
}

\author{
Martin J. Meyer, Martin A. Zwaan, Rachel L. Webster \\ School of Physics, University of Melbourne, VIC 3010, Australia \\ Stephen E. Schneider \\ Department of Astronomy, University of Massachusetts, Amherst, MA \\ 01003, USA
}

\begin{abstract}
The Tully-Fisher relation is of interest because of its use as a secondary distance measure and the constraints it places on the physics of rotationally supported galaxies. We use data from the HI Parkes AllSky Survey Catalogue to study and apply the Tully-Fisher relation on a sample of galaxies selected on their HI properties. The issues of third parameter dependencies and intrinsic scatter of the Tully-Fisher relation are investigated.
\end{abstract}

\section{Introduction}

The physical origins of the Tully-Fisher relation are yet to be fully understood. It is still unclear why the scatter in the luminosity-linewidth relation is so small, and hence why the star formation histories of spiral galaxies are so well determined solely by their rotational velocities.

The HI Parkes All-Sky Survey Catalogue (HICAT) removes Tully-Fisher biases introduced by the optical pre-selection of galaxies for which HI profiles are then obtained. We use this sample to examine relational dependencies on properties such as environment, surface brightness, morphology and HI profile shape. Furthermore, by applying cuts to parameters that are physically independent of relational scatter, such as galaxy inclination, the fundamental issue of intrinsic scatter is addressed.

\section{Data}

HICAT was constructed using data from the southern region of the HI Parkes All-Sky Survey (HIPASS), a blind HI survey with the Parkes radio telescope covering the entire sky $\delta<+25^{\circ}$. In total, 4,315 sources were recovered with declinations $\delta<+2^{\circ}$ over the velocity range 300 to $12,700 \mathrm{~km} \mathrm{~s}^{-1}$ (Meyer et al. 2003). This catalogue is $\sim 16$ times the size of any blind HI catalogue compiied prior to HIPASS. 2MASS Extended Source Catalogue (Jarrett et al. 2000) $K$ band magnitudes are used to provide luminosity data, with $~ 75 \%$ of HIPASS detections having a counterpart within $3 \sigma$. Observed quantities are corrected 

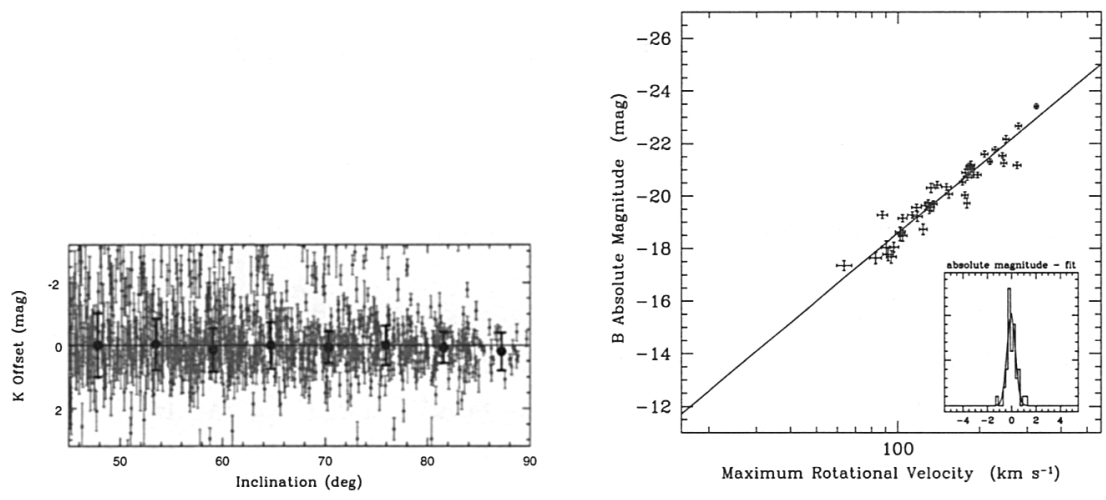

Figure 1. (left) Absolute magnitude minus Tully-Fisher fit as a function of galaxy inclination. (right) Tully-Fisher relation with tight galaxy selection cuts based on observational parameters.

to allow for effects such as extinction, galaxy inclination and turbulent motions. Luminosities are calculated assuming Hubble distances.

\section{Parameter Dependencies and Intrinsic Scatter}

Dependencies are found on a number of observational parameters including galaxy inclination (see left panel Figure 1), apparent size, angle from the Cosmic Microwave Background (CMB) dipole and velocity. The dependence on CMB dipole angle is the result of local bulk flows, with nearby galaxies at rest with respect to the Local Group, but the velocities of more distant galaxies converging with that of the CMB. Examining intrinsic parameters, potential dependencies are found for $(B-R)$ colour and far-infrared (FIR) luminosity. The sense of the correlation is for galaxies with the highest FIR luminosities to lie above the Tully-Fisher relation, consistent with these galaxies currently undergoing more intense star formation.

To assess the intrinsic scatter of the Tully-Fisher relation, cuts are applied to purely observational parameters. Shown in the right-hand panel of Figure 1 is the resultant Tully-Fisher relation for galaxies having: inclination $>75^{\circ}$, size $>30^{\prime \prime}$ (optical major axis), angle from the CMB dipole equator $\theta_{C M B}<20^{\circ}$ and velocity $>1000 \mathrm{~km} \mathrm{~s}^{-1}$. The scatter of this relation is $0.31 \mathrm{mag}$. Allowing for measurement errors, this corresponds to an intrinsic scatter of $0.24 \mathrm{mag}$.

\section{References}

Jarrett, T. H., Chester T., Cutri, R., Schneider, S., Skrutskie, M., \& Huchra, J. P. 2000, AJ, 119, 2498

Meyer, M.J., Zwaan, M.A., Webster, R.L., Staveley-Smith, L., et al. 2003, MNRAS, submitted. 